\title{
Aspectos subjetivos e representacionais do viver com HIV: o que as publicações revelam?
}

\section{Subjective and representational aspects of living with HIV: what do the publications reveal?}

\author{
Renata Lacerda Marques Stefaisk' $\bullet$ Denize Cristina de Oliveira² ${ }^{2}$ Sergio Corrêa Marques ${ }^{3}$ \\ Yndira Yta Machado ${ }^{4}$
}

\begin{abstract}
RESUMO
O presente estudo teve como objetivo identificar, na literatura nacional e internacional, aspectos subjetivos do viver com HIV presentes nas produções científicas acerca do HIVIAIDS, apoiados na Teoria das Representações Sociais. Realizou-se uma revisão sistemática de artigos científicos indexados nas bases de dados LILACS, BDENF e MEDLINE, utilizando os seguintes descritores: HIV AND AIDS AND Representação Social. Dos II 8 artigos encontrados, 8 atenderam os critérios de inclusão para análise. Os itens relativos aos resultados e as discussões dos estudos selecionados foram submetidos à análise de conteúdo temático-categorial. Foram identificadas 220 unidades de registro, distribuídas entre 5 categorias: Informação e prevenção do HIVIAIDS; Preconceito e estigmatização vivenciados pelas pessoas que vivem com HIV; Sofrimento trazido pelo HIV; Construção e mudança da representação social da AIDS; Qualidade de vida e estruturas de apoio. Conclui-se que o conhecimento acerca dos aspectos subjetivos e representacionais do HIV e a valorização do conhecimento do senso comum são fundamentais para direcionar novas estratégias de saúde com o intuito de prover uma atenção mais integral às pessoas que vivem com o agravo.
\end{abstract}

Palavras Chave: HIV;AIDS; Representação social; Preconceito.

\begin{abstract}
The present study aimed to identify, in the national and international literature, subjective aspects of living with HIV present in scientific productions about HIVIAIDS, supported by the social representations theory. An sistematic review of scientific articles indexed in databases LILACS, BDENF and MEDLINE was carried out, using the following descriptors: HIV and AIDS and Social Representations. Of I 18 articles found, 08 were included because they met the inclusion criteria. The results and discussions of the selected studies were submitted to the thematic-categorial content analysis. A total of 220 registration units were identified, distributed among 5 categories: HIV/AIDS information and prevention; Prejudice and stigmatization experienced by people living with HIV; Suffering brought on by HIV; Building and changing the social representation of AIDS; Quality of life and support structures. It is concluded that knowledge about the subjective and representational aspects of HIV and the valorization of common-sense knowledge are fundamental to direct new health strategies in order to provide an integral attention to the people living with the disease.
\end{abstract}

Keywords: HIV;AIDS; Social Representation; Prejudice.

I. Enfermeira. Doutoranda pelo Programa de Pós-graduação em Enfermagem da Universidade do Estado do Rio de Janeiro - UERJ. Rio de Janeiro, Brasil. E-mail: renata_350@hotmail.com ORCID ID: https://orcid.org/0000-000I-5592-7565

2. Professora Adjunta no Departamento de Fundamentos de Enfermagem e no Programa de Pós-Graduação em Enfermagem da Faculdade de Enfermagem da Universidade do Estado do Rio de Janeiro - UERJ. Rio de Janeiro, Brasil. E-mail: dcouerj@gmail.com ORCID ID: https://orcid.org/0000-0002-0830-0935.

3. Enfermeiro. Professor Adjunto no Departamento de Fundamentos de Enfermagem e no Programa de Pós-Graduação em Enfermagem da Faculdade de Enfermagem da Universidade do Estado do Rio de Janeiro - UERJ. Rio de Janeiro, Brasil. E-mail: sergiocmarques@uol.com.br ORCID ID: https://orcid.org/0000-0002-0038-0790.

4. Enfermeira. Doutoranda pelo Programa de Pós-graduação em Enfermagem da Universidade do Estado do Rio de Janeiro - UERJ. Rio de Janeiro, Brasil. Bolsista Nota I0 FAPERJ. E-mail:yndiramachado@gmail.com ORCID ID: https://orcid.org/0000-0003-0692-7299. 


\section{INTRODUÇÃO}

A síndrome da imunodeficiência adquirida (AIDS) no Brasil despertou a atenção internacional devido ao número de casos notificados, à complexidade da epidemia e à atuação do governamental em prol do acesso universal e gratuito aos medicamentos, fato que influenciou fortemente o curso da doença ${ }^{(1)}$. Entre 1980 e junho de 2018, foram notificados 926.618 casos de AIDS no Brasil, sendo que o país tem registrado uma média anual de 40 mil casos da doença. Até junho de 2017, 20,9 milhões de pessoas vivendo com HIV tiveram acesso à terapia antirretroviral (TARV) ${ }^{(2)}$, dado que evidencia um crescimento significativo do acesso ao tratamento ${ }^{(3)}$.

Diante da prevalência da doença, a prevenção e o controle do vírus têm sido um desafio para a área da saúde nas últimas décadas, uma vez que a pandemia gerada pelo HIV é de natureza complexa ${ }^{(4)}$. Acrescenta-se que a necessidade do conhecimento das condições psicossociais atreladas às pessoas vivendo com HIV e as suas relações com a doença têm importância para a abordagem destas no contexto da atenção à saúde, fundamentando, então, a necessidade dos estudos sobre representações sociais acerca do $\mathrm{HIV}^{(5)}$.

A epidemia da AIDS evidenciou que o processo saúde-doença é mediado por determinantes e condicionantes que vão desde a consideração do indivíduo enquanto sujeito de intersubjetividade, até a sociedade como um todo(6). Dessa forma, é importante conhecer os aspectos subjetivos e de cunho psicossocial que influenciam o viver com HIV de modo a colaborar para a construção de estratégias que promovam uma atenção mais direcionada para as reais necessidades de saúde dessa população. Nesse sentido, a Teoria das Representações Sociais traz um caminho para a compreensão do saber popular e coletivo desenvolvido acerca dessa síndrome, bem como sua influência sobre as práticas do grupo que vive com HIV.

As representações sociais são conceituadas como "uma modalidade de conhecimento particular que tem por função a elaboração de comportamentos e a comunicação entre indivíduos no quadro da vida cotidiana"(7). Uma de suas funções é permitir o acesso ao conhecimento social que orienta as práticas de um determinado grupo quanto à problemas de saúde, ou seja, identificar o conhecimento que o grupo utiliza para interpretar seus problemas e justificar suas práticas sociais ${ }^{(8)}$.

Desta forma, faz-se necessário maior divulgação do conhecimento construído por meio de estudos de revisão que contribuam para a difusão da produção científica acerca do assunto. Acrescenta-se que discutir os aspectos psicossociais da AIDS abordados a partir das representações sociais é fundamental para compreender a configuração simbólica do fenômeno e os modos de pensar e vivenciar a doença, tornando possível a elaboração de estratégias que gerem melhoria na qualidade de vida desse grupo.

Nesse contexto, o questionamento que norteou este estudo foi: quais os aspectos subjetivos do viver com AIDS abordados nas produções científicas acerca do HIV/AIDS e suas representações sociais, divulgadas em periódicos científicos? O objetivo deste estudo é identificar, na literatura nacional e internacional, aspectos subjetivos e representacionais do viver com HIVIAIDS presentes nas produções científicas sobre o tema.

\section{MÉTODO}

Trata-se de revisão de literatura do tipo sistemática, com abordagem qualitativa e descritiva. A revisão sistemática é particularmente útil para integrar as informações de um conjunto de estudos realizados separadamente e também para identificar a necessidade de futuras pesquisas diante de resultados conflitantes ou não evidenciados ${ }^{(9)}$.

O percurso seguido para a realização desta revisão sistemática incluiu as etapas: elaboração da pergunta de pesquisa; busca na literatura; seleção dos artigos; extração dos dados; avaliação da qualidade metodológica; síntese dos dados; avaliação da qualidade das evidências e redação e publicação dos resultados ${ }^{(10)}$.

A primeira etapa da investigação foi delineada a partir do questionamento de estudo. Para selecionar a amostra de publicações foi realizada busca de literatura nas bases de dados Scientific Eletronic Library Online (LILACS), Base de Dados de Enfermagem (BDENF) e Medical Literature Analysis e Retrieval System Online (MEDLINE), sendo adotados os seguintes critérios de inclusão: artigos gratuitos e disponíveis on-line na íntegra, nos idiomas português, inglês e espanhol e que abordassem as representações sociais e o HIVIAIDS. Com a intenção de captar o maior número de artigos possível que respondessem à questão de pesquisa, não foi estabelecido recorte temporal. Foram adotados como critérios de exclusão teses e dissertações, publicações com duplicidade na base de dados consultada ou cujo tema não se relacionava ao recorte proposto.

Os dados foram coletados no mês de setembro de 2017 , mediante busca nas referidas bases de dados. Para tanto, utilizou-se os seguintes descritores e combinações: "HIV AND AIDS AND Representação Social".

Foram identificados II4 artigos na primeira etapa da busca; após aplicação dos critérios de exclusão restaram 36 publicações, as quais passaram por um processo de leitura dos seus títulos e resumos. A amostra final foi constituída por 8 publicações que atenderam aos critérios de inclusão.

Os itens relativos aos resultados e as discussões dos estudos selecionados foram submetidos à análise de con- 
teúdo temático-categorial. $\mathrm{A}$ análise de conteúdo pode ser definida como um conjunto de técnicas de análise das comunicações que visa obter, por meio de procedimentos sistemáticos e objetivos de descrição do conteúdo das mensagens, indicadores que permitam a inferência de conhecimentos relativos ao conteúdo das mensagens ${ }^{(I)}$.

A análise de conteúdo pode ser compreendida como um processo através do qual o material empírico é cuidadosamente transformado, de forma sistemática, e codificado em unidades, as quais permitem uma descrição exata das características pertinentes ao conteúdo dos discursos analisados 12. O tipo de análise de conteúdo empregado neste estudo foi o da análise temática, que consiste em descobrir os núcleos de sentido que compõem uma comunicação, cuja presença ou repetição seja relevante ao objetivo do estudo.

Os procedimentos necessários para a realização da análise de conteúdo temático-categorial, foram: I. leitura flutuante, intuitiva, ou parcialmente orientada do texto; 2 . definição de hipóteses provisórias sobre o objeto estudado e o texto analisado; 3 . determinação das unidades de registro (UR); 4. marcação no texto do início e final de cada UR observada; 5 . definição das unidades de significação (US) ou temas; 6. análise temática das US; 7. análise categorial do texto; 8. tratamento e apresentação dos resultados ${ }^{(12)}$.

\section{RESULTADOS E DISCUSSÃO}

Após seleção dos artigos foram incluídos nesta análise os oito textos constantes no quadro I, que sintetiza os artigos encontrados e a apresenta informações sobre os autores, título, ano de publicação e identificação do periódico de origem.

Destaca-se que cinco textos foram veiculados em periódicos do campo da saúde e outros três da Psicologia, o que demonstra a pertinência da teoria de representações sociais ao campo da Psicologia e sua utilização destacada no campo da saúde, conforme apontado por diversos estudos. Quanto a distribuição temporal, observa-se dois estudos nos anos de 201 I e 2014, um nos demais anos, nenhuma publicação no ano de 2017.

No que se refere ao periódico de veiculação do artigo, dois foram publicados na Revista Latinoamericana de Enfermagem e um em cada um dos seguintes periódicos: Caderno de Saúde Pública; Revista Enfermagem UERJ; Psicologia, Teoria e Prática; Psicologia e Sociedade; Psicologia em Estudo; Revista da Escola de Enfermagem USP.

Uma vez que as representações sociais conjugam a capacidade de perceber e compreender o cotidiano de modo a fornecer um sentido às situações vivenciadas pelo grupo, depreende-se que os resultados dos estudos analisados refletem as crenças das pessoas que vivem com HIV, ou seja, sua representação acerca do fenômeno AIDS.

Como resultado da análise de conteúdo temático-categorial foram construídas cinco categorias que revelam os conteúdos veiculados pelos estudos analisados nas sessões de resultados e discussões. Foram recortadas 220 unidades de registro (UR), distribuídas da seguinte forma: I - Informação e transmissão do HIVIAIDS (6I UR); 2 - Preconceito e estigmatização vivenciados pe-

QUADRO 1 - Estudos sobre representações sociais e HIVIAIDS. 2019

\begin{tabular}{|l|l|c|l|}
\hline Título do artigo & Autores & Ano de Publicação & Periódico (vol, $\mathrm{n}^{\circ}$, pág, ano) \\
\hline $\begin{array}{l}\text { Aids and jail: social representations of women } \\
\text { in freedom deprivation situations }\end{array}$ & $\begin{array}{l}\text { TRIGUEIRO, D. R. S. G.; ALMEIDA, S. } \\
\text { A.; MONROE, A. P.; COSTA, G. P. O.; } \\
\text { BEZERRA, V. P.; NOGUEIRA, J. A. }\end{array}$ & 2016 & $\begin{array}{l}\text { Rev Esc Enferm USP. } \\
\text { v.50, n.4. 2016. }\end{array}$ \\
\hline $\begin{array}{l}\text { Qualidade de vida e AIDS sob a ótica de } \\
\text { pessoas vivendo com o agravo: contribuição } \\
\text { preliminar da abordagem estrutural das } \\
\text { representações sociais }\end{array}$ & $\begin{array}{l}\text { COSTA, T. L.; OLIVEIRA, D. C. O.; } \\
\text { FORMOZO,_G. A. }\end{array}$ & 2015 & $\begin{array}{l}\text { Cad Saúde Pública, v.31, } \\
\text { n.2. p. 365-376, 2015. }\end{array}$ \\
\hline $\begin{array}{l}\text { Representações sociais e estereótipos sobre } \\
\text { aids e pessoas que vivem com HIVIAids }\end{array}$ & $\begin{array}{l}\text { ANTUNES, L.; CAMARGO, B. V.; } \\
\text { BOUSFIELD, A. B. S. }\end{array}$ & 2014 & $\begin{array}{l}\text { Psicol. teor. prát; v.16, n. 3, } \\
p .43-57,2014 .\end{array}$ \\
\hline $\begin{array}{l}\text { Representações sociais de caminhoneiros de } \\
\text { rota curta sobre HIVIAIDS }\end{array}$ & $\begin{array}{l}\text { SOUSA, L. M. S. S., LUCINEIDE S.; } \\
\text { PALMEIRA, A. T. }\end{array}$ & 2014 & $\begin{array}{l}\text { Psicol. soc. (Online); v.26, } \\
n .2 . p .346-355,2014 .\end{array}$ \\
\hline $\begin{array}{l}\text { Persons living with AIDS in nurses' social } \\
\text { representations: analysis of central, } \\
\text { contranormative and attitudinal elements }\end{array}$ & $\begin{array}{l}\text { COSTA, T. L.; OLIVEIRA, D. C. O.; } \\
\text { FORMOZO, G. A. GOMES, A. M. T. }\end{array}$ & $\begin{array}{l}\text { Rev Lat Am Enfermagem; } \\
\text { v.20, n. 6, } p .1091-1099, \\
2012 .\end{array}$ \\
\hline $\begin{array}{l}\text { O significado do HIVIAIDS no processo de } \\
\text { envelhecimento }\end{array}$ & $\begin{array}{l}\text { OLIVEIRA, D. C.; OLIVEIRA, E. G.; } \\
\text { GOMES, A. M. T.; TEOTÔNIO, M. C.; } \\
\text { WOLTER, R. M. C. P. }\end{array}$ & 2012 \\
\hline $\begin{array}{l}\text { Social representations of AIDS and their } \\
\text { quotidian interfaces for people living with HIV }\end{array}$ & $\begin{array}{l}\text { GOMES, A. M. T.; SILVA, E. M. P.; } \\
\text { OLIVEIRA, D. C. }\end{array}$ & 2011 & $\begin{array}{l}\text { Rev. enferm. UERJ; v.19, } \\
n .3 . p .353-358,2011 .\end{array}$ \\
\hline $\begin{array}{l}\text { AIDS, relações conjugais e confiança: um } \\
\text { estudo sobre representações sociais }\end{array}$ & OLTRAMARI, L. C.; CAMARGO, B. V. & $\begin{array}{l}\text { Rev Lat Am Enfermagem; } \\
\text { v.19, n.3, p. 485-492, 2011. }\end{array}$ \\
\hline
\end{tabular}


las pessoas que vivem com HIV (45 UR); 3 - Sofrimento trazido pelo HIV (42 UR); 4 - Construção e mudança da representação social da AIDS (40 UR); 5 - Qualidade de vida e estruturas de apoio (32 UR). Essas categorias serão apresentadas a seguir.

\section{I - Informação e transmissão do HIVIAIDS}

Esta categoria foi, quantitativamente, a mais expressiva da análise, apresentando $27,8 \%$ do total das 220 UR. Ela englobou os aspectos relativos à difusão de informação acerca do HIV e sua influência sobre o comportamento sexual dos indivíduos.

Alguns exemplos de UR retiradas dos estudos analisados demonstram essa abordagem:

A falta de informação sobre a AIDS pode gerar concepções errôneas das formas de transmissão do vírus [...] $O$ comportamento dos adolescentes está associado à qualidade das informações que possuem ${ }^{(13)}$.

Os mais jovens estavam mais sensibilizados com a ideia de prevenção, sobretudo em decorrência do apelo ao uso do preservativo, evidenciado desde a sua juventude até os dias atuais(6).

Obter informações concretas sobre o HIVIAIDS, quanto aos meios de transmissão e de prevenção favorece, de forma positiva, no enfrentamento da epidemia ${ }^{(16)}$.

Estes sujeitos puderam assumir certa negação do risco ao HIV, o que foi encontrado principalmente quando indagados a respeito da possibilidade de infecção deles pelo vírus(6).

Os trechos destacados apontam para o impacto da informação científica sobre as práticas associadas à doença. Sob esse aspecto, observa-se que, apesar das taxas de informação acerca do HIV terem aumentado, apenas $36 \%$ de homens jovens e 30\% de mulheres jovens (entre I5-24 anos) possuem um conhecimento abrangente e correto sobre como prevenir o HIV em 37 países estudados ${ }^{(2)}$.

Considerando-se o grave problema que a infecção pelo HIV representa para a saúde pública, torna-se especialmente relevante colocar em prática estratégias de prevenção por meio da educação em saúde, as quais divulguem os conhecimentos necessários para a compreensão do vírus e realização de medidas de prevenção. Nesse contexto, um projeto continuado de sensibilização para promoção da saúde se torna estratégico, devido ao efeito multiplicador e disseminador das informações nas comunidades de origem ${ }^{(17)}$.

Apesar do investimento no sentido de disseminar informações relativas à doença e seus modos de contágio, observou-se nas publicações uma tendência de desvalorização do risco da contaminação, associando-a apenas às relações com parceiros casuais, o que pode ser observado em uma das UR destacadas ${ }^{(6)}$.

Sob esse aspecto, considera-se que a vulnerabilidade para a aquisição do HIV entre adolescentes e jovens é maior quando a transição de idade ocorre em ambientes menos favorecidos, com acesso insuficiente à alimentação, educação e moradia e com altas taxas de violência. Dessa forma, percepção de baixa exposição a infecção, uso descontínuo de preservativo e baixas taxas de testagem de HIV persistem entre os jovens ${ }^{(2)}$.

No que se refere aos conhecimentos e informações que o grupo detém acerca da AIDS, torna-se relevante acrescentar que eles participam da construção das ideias e concepções de mundo desses sujeitos, ou seja, constituem suas representações acerca do fenômeno. Sob esse aspecto, considera-se que a motivação para a elaboração da representação social é a tentativa de "construir uma ponte entre o estranho e o familiar"(18).

\section{2 - Preconceito e estigmatização vivenciados pe- las pessoas que vivem com HIV}

A segunda maior categoria apresentou $45 \mathrm{UR}$, as quais correspondem a $20,5 \%$ do total e incluiu aspectos relativos ao preconceito e à estigmatização vivenciados pelas pessoas vivendo com HIV. Observa-se que esses são aspectos sensíveis para esse grupo e que o medo da discriminação e da rejeição, inclusive no contexto das relações afetivas, frequentemente leva o indivíduo a não revelar sua condição sorológica ${ }^{(13,16,19)}$.

Destacam-se as UR retiradas dos estudos analisados:

Atos discriminatórios para com as pessoas que vivem com HIVIAIDS estão relacionados com comportamentos de distância social, com a atribuição de responsabilidade e de estereótipos, constituindo-se em obstáculos para a convivência social(20).

Quando um determinado grupo sofre discriminação, abuso e repressão, existe uma maior chance de não procurar testagem, prevenção ou serviço de tratamento para AIDS ${ }^{(14)}$.

As pessoas soropositivas apresentam, como estratégias de sobrevivência social, o ocultamento da doença. Assim, podem continuar a vida como pessoas normais, sem serem acusadas e discriminadas, seja no âmbito familiar, social ou no trabalho( ${ }^{(16)}$.

A atribuição de responsabilidade e culpabilização dessas pessoas pode ser verificada neste estudo, sendo um elemento presente nas representações sociais do grupo estudado ${ }^{(20)}$.

As diferentes formas de estigma e discriminação em relação as pessoas que vivem com HIV têm ocorrido desde a construção das primeiras representações da AIDS, devido às características dos primeiros casos da doença. Elas geram o isolamento do grupo e impactam nas vidas desses sujeitos, gerando inclusive auto estigmatização, por meio da qual os indivíduos tendem a interiorizar as crenças sociais negativas e passar a evitar o contato social|(23).

A presença do preconceito nas vivências reais ou imaginárias desse grupo revela a sua persistência como um significado remanescente da representação social da AIDS desde o início da epidemia ${ }^{(24)}$. Esse elemento expressa um aspecto fundamental para uma abordagem 
psicossocial das pessoas que vivem com HIV devido ao seu impacto simbólico e prático, uma vez que o conceito está arraigado à própria vivência da soropositividade e que a discriminação afeta profundamente a vida dessas pessoas, causando sensação de sofrimento e pesar ${ }^{(19)}$.

O preconceito determina também o afastamento familiar e laboral, uma vez que frequentemente existem dificuldades para compartilhamento do diagnóstico com outras pessoas, especialmente aquelas com quem existe a possibilidade de maior envolvimento afetivo(21). Adicionalmente, ele implica, entre os portadores do vírus em não aceitação do diagnóstico e abandono do tratamento, interferindo diretamente na qualidade de vida desses indivíduos ${ }^{(22)}$.

Num contexto de persistência de ideias de cunho discriminatório e excludente, a culpabilização emerge como uma estratégia disciplinar de modelamento de subjetividades. Dessa forma, a efetivação de um discurso que culpabiliza os sujeitos soropositivos é fruto de um clamor moralizante que almeja o controle das ações individuais e de grupos específicos ${ }^{(25)}$.

\section{3 - Sofrimento trazido pelo HIV}

Esta categoria apresentou 42 UR, as quais correspondem a $19,1 \%$ do total e incluiu aspectos relativos ao sofrimento trazido pela confirmação do diagnóstico. Essas situações de sofrimento, inclusive pela associação da AIDS à uma doença mortal, podem ser observados nos exemplos de UR retirados dos estudos analisados:

Outro elemento da representação social da AIDS é o de doença como sendo ruim, [...] e tal resultado sugere uma associação ao sofrimento fisico (emagrecimento, déficit na aparência visual, invalidez, dentre outros) e psíquico (preconceito, depressão, estar com uma doença incurável) $)^{(6)}$.

O sofrimento não é só em decorrência do processo de adoecimento, mas está relacionado também as faces da AIDS, a partir das quais se entende a cicatriz social produzida pelo agravo(14).

A pessoa que vive com HIVIAIDS é vista como alguém condenado ao sofrimento e à morte, ao isolamento e à tristeza, já que a AIDS é vista como uma sentença de morte tanto fisica quanto social (20).

Os trechos destacados evidenciam uma forte associação da AIDS com a morte e o sofrimento, seja devido aos desdobramentos físicos da doença ou às influências psicológicas e subjetivas trazidas por uma doença potencialmente fatal. Sob esse aspecto, acrescenta-se que viver com HIV gera profundas mudanças na saúde emocional do indivíduo, uma vez que o mesmo se vê diante da possibilidade de perder saúde, família, trabalho e até mesmo o controle de suas próprias escolhas, o que pode comprometer a autoestima e gerar impactos em todas as áreas da vida ${ }^{(26)}$.
A descoberta da soropositividade frequentemente acarreta uma ruptura dos sonhos que, anteriormente ao diagnóstico se expressavam em perspectivas de futuro. Sob esse aspecto, muitos sujeitos que vivem com o vírus relatam que antes de receber o diagnóstico sentiam-se mais aptos a desenvolver suas atividades diárias e planejar seu futuro(22).

Dessa forma, depreende-se que o medo, frequentemente, é um sentimento comum as pessoas com HIV, os quais relatam temer o preconceito, $\circ$ afastamento das pessoas e a possibilidade de perder o respeito das mesmas ${ }^{(22)}$. Sobre esse aspecto, acrescenta-se que a vivência da soropositividade vem acompanhada de condições que favorecem o sofrimento ou adoecimento psíquico, as quais podem ser desencadeadas por sentimentos contraditórios que surgem diante da tentativa de evitar algo que causa dor ${ }^{(26)}$.

\section{4 - Construção e mudança da representação so- cial da AIDS}

Esta categoria apresentou $40 \mathrm{UR}$, as quais correspondem a $18,1 \%$ do total e incluiu aspectos relativos à construção e às mudanças que ocorreram na representação social da AIDS nos últimos anos. Esse fato pode ser observado nos trechos em destaque a seguir:

AIDS estava associada a [...] doença incurável por sua relação com a morte. Tais representações estão ancoradas historicamente na construção social da AIDS, que traz, desde a sua origem, a relação com o sofrimento e a finitude ${ }^{(6)}$.

Na formação das RS da AIDS, as influências da mídia, as descobertas embrionárias da ciência e o conhecimento médico ainda incipiente do princípio da epidemia determinaram estereótipos atrelados à pessoa que vive com HIVIAIDS (14).

Outro processo de ancoragem presente nas representações do grupo reafirma a ligação da AIDS com a diabetes, configurando a síndrome como uma doença crônica, não fatal e que possui controle através de fármacos disponíveis por programas governamentais ${ }^{(16)}$.

O início do advento da AIDS trouxe à tona o medo de uma doença grave e fatal que não era plenamente compreendida, assim, essa lacuna de conhecimento foi preenchida pelos discursos de diversas instituições como Estado, medicina e igreja ${ }^{(27)}$. Nesse contexto de inicial desconhecimento, as impressões sobre os modos de contágio da AIDS foram associadas a aspectos morais e imagens de transgressão os quais foram maciçamente difundidos pela mídia e assimilados pela população, gerando uma representação negativa ${ }^{(28)}$.

O desenvolvimento do conhecimento que trouxe à tona a compreensão acerca da patogênese do HIV e seus desdobramentos, como o desenvolvimento da terapia antirretroviral de alta potência (TARV), foram fatores fundamentais para a mudança da sua represen- 
tação. Dessa forma, o aumento da sobrevida por portadores de HIV acarretou transformações no plano simbólico da representação, tornando secundária a associação AIDS/morte ${ }^{(24)}$.

Acrescenta-se que a conquista do acesso ao tratamento, a maior adesão à terapia antirretroviral, a ampliação da oferta do diagnóstico e a cronicidade da doença têm provocado queda da morbimortalidade, aumento da expectativa de vida e ressignificação de projetos futu$\operatorname{ros}^{(29)}$. Esses fatores, somados à efetivação de medidas de enfrentamento da AIDS, têm influenciado uma mudança representacional, no sentido da incorporação do significado de doença crônica o qual vem sendo assumido pela AIDS nos últimos anos ${ }^{(30)}$. Destaca-se que esse movimento de mudança da representação da AIDS é marcado pelo desenvolvimento de medidas de enfrentamento da doença e pela percepção da necessidade de prevenção do contágio. De fato, da segunda metade dos anos 2000 e início de 2010 foi um período marcado pela construção da "AIDS como doença crônica", passando a abarcar, no núcleo central da representação social, significados positivos e elementos normativos de enfrentamento da doença (a exemplo de tratamento, prevenção e cuidado) ${ }^{(24)}$.

Sob esse aspecto, destaca-se o processo de afastamento da cognição morte dos elementos organizadores da representação da AIDS, fato que permitiu a introdução de outros com conotação mais positiva e que passaram a ocupar o lugar do desfecho fatal inicialmente atrelado à doença ${ }^{(13)}$. Tal mudança reflete uma melhoria da qualidade de vida desse grupo, abrindo espaço para discussões relativas à adaptação, estratégias de enfrentamento e ressignificação de vida.

\section{5 - Qualidade de vida e estruturas de apoio social}

Esta categoria apresentou 32 UR, as quais correspondem a $14,5 \%$ do total e incluiu aspectos relativos à perspectiva da qualidade de vida entre as pessoas que vivem com HIV e também a importância das estruturas de apoio para a manutenção da integração social desses indivíduos. Acerca desses aspectos, destacam-se os trechos abaixo:

$O$ desejo de viver e de aproveitar a vida ainda são preservados, originando sentimentos de esperança. Os sujeitos afirmam buscar aproveitar o tempo com ocupações e distrações, além de construir uma forma particular de conviver com o HIVIAIDS(15).

Quanto ao componente trabalho, o conjunto das evocações abarca a sua concepção como trabalho em si, sendo atividade produtiva, bem como as facetas de empregabilidade e oportunidade no mercado como algumas de suas possíveis repercussões psicossociais ${ }^{(13)}$.

No que tange à posição dos sujeitos sobre o papel da família para as pessoas com HIVIAIDS, essa representa importante espaço de superação de carências emocionais elou sociais(31).

O conviver com HIVIAIDS [...] estrutura-se na interface da pluralidade e da complexidade do funcionamento de elementos comportamentais em busca de viver com qualidade, mesmo diante da cronicidade e da incerteza do diagnóstico(15).

[O sucesso do tratamento] depende, sobretudo, de disciplina e exige alguns cuidados, o que os estimula a incorporar hábitos saudáveis na rotina diária, como dormir cedo, se alimentar bem, fazer exercício fisico, não beber, não fumar e manter alimentação balanceada ${ }^{(16)}$.

Diante de um contexto em que o viver com AIDS passou a ocupar um lugar central que antes era suprimido pelo medo de morrer devido à doença, as estruturas de apoio emergem como uma forma de promover maior ajuste das pessoas que vivem com HIV no retorno às suas atividades da vida diária, com destaque para a família, que frequentemente se configura como o alicerce desses indivíduos. Observa-se que o apoio dos familiares é capaz de mudar o curso do enfrentamento da doença por meio da reestruturação e aceitação de si mesmo, depois do impacto trazido pelo diagnóstico(22).

Dessa forma, ao enfrentarem a doença, esse grupo realiza mudanças comportamentais e na sua rotina da vida diária, as quais muitas vezes resultam em uma qualidade de vida melhor do que aquela vivenciada antes do diagnóstico. Sob esse aspecto, destacam-se os trechos que abordaram as mudanças realizadas na rotina de vida após o diagnóstico. De fato, buscar a convivência com o HIV acarretou mudanças comportamentais, com maior atenção à alimentação e atividade física e incorporação de hábitos de vida mais saudáveis.

Acrescenta-se que os avanços no conhecimento da doença possibilitaram manejo clínico e trouxeram meIhorias na qualidade de vida desse grupo, trazendo avanços na compreensão da doença que geraram aumento da sobrevida e, em contrapartida, trazendo à tona outras facetas do processo saúde-doença, como os desafios psicossociais do viver com $\operatorname{AIDS}^{(13)}$.

\section{CONCLUSÃO}

Os resultados deste estudo destacaram alguns aspectos da representação social da AIDS evidenciados pelas publicações. Dessa forma, os significados e as crenças atrelados à doença se referiram à importância da divulgação de informações científicas sobre a AIDS e, também, à necessidade de valorização do senso comum. Acrescenta-se que a discriminação, o estigma e o sofrimento trazidos pela doença são constitutivos da representação social da AIDS e decorrem, em parte, da memória social construída da doença ao longo das décadas, a qual persiste como um significado remanescente presente nas representações da doença nos dias atuais. 
O desenvolvimento do conhecimento científico e a disponibilização de tratamentos emergem como elementos desencadeadores de mudança da representação da AIDS, de modo que parte do grupo que vive com HIV passou a percebê-la não como uma sentença de morte, mas como uma doença crônica que traz consigo possibilidades de convivência. A discussão da qualidade de vida, por sua vez, surgiu como um desdobramento dessa mudança, a qual acarretou o desejo de viver e a construção de perspectivas de futuro como formas de enfrentamento da doença.

A presente pesquisa não esgota a possibilidade de futuras contribuições acerca da temática, porém, espera- se que, por meio do conhecimento das representações sociais da AIDS e do saber construído pelo grupo acerca desse fenômeno seja possível contribuir de forma positiva para o conhecimento acerca dos aspectos subjetivos de viver com HIV e direcionar novas estratégias de saúde com o intuito de prover uma atenção mais integral às pessoas que vivem com a síndrome.

Destaca-se como limitação deste estudo a possível heterogeneidade das publicações incluídas na revisão e o fato da palavra chave representação social não fazer parte do DeCS, dificultando a localização dos estudos orientados por esse referencial teórico. 


\section{REFERÊNCIAS}

I. Sousa CFO, Silva, AL. O cuidado a pessoas com HIVIAIDS na perspectiva de profissionais de saúde. Rev. Esc. Enferm. USP. 20। 3; 47(4): 907-9।4.

2. UNAIDS. Joint United Nations Programme on Hiv/AIDS. World Health Organization. [internet]. 2017. [Acesso em: 22 jul 20I7]; p.6. Disponível em: http://unAIDS.org. $\mathrm{br} / 2017 / 07 / 19-\mathrm{mi}-$-em-tratamento-hiv-mortes-relacionadas -AIDS-caem-no-mundo/

3. Ministério da Saúde (BR). Boletim Epidemiológico HIV/ AIDS. Brasília: DF. 2018. 49(53).

4. Okuno MFP et al. Quality of life in elderly patients living with HIVIAIDS. Cad. Saúde Pública. 20 I4; 30(7): I 55 I-59.

5. Dantas MS et al. Representações Sociais do HIVIAIDS por profissionais de saúde em serviços de referência. Rev. Gaúcha Enferm. 20।4. 35(4):94-100.

6. Sousa LMS, Silva LS, Palmeira AT. Representações sociais de caminhoneiros de rota curta sobre HIVIAIDS. Psicol. Soc. 2014. 26(2): 346-55.

7. Moscovici, S. A representação social da psicanálise. Tradução de Cabral. Rio de Janeiro: Zahar; 1978.

8. Oliveira DC.A teoria de representações sociais como grade de leitura da saúde e da doença: a constituição de um campo interdisciplinar. In: A.M.O. Almeida MFS, Santos ZA, Trindade (Orgs.) Teoria das representações sociais 50 anos. Brasília:Technopolitik; 20I4. p. 775-8I4.

9. Figueiredo MLF, Silva MHR, Mendes WC. Revisão Sistemática: um caminho para evidências na produção científica de enfermagem. Revista Saúde em Foco. 2014. I(I): 72-8I.

10. Galvão TF, Pereira MG. Revisões sistemáticas da literatura: passos para sua elaboração. Epidemiol. Serv. Saúde. 2014. 23(I): 183-184.

I I. Bardin, L. Análise de conteúdo. Lisboa: Edições 70; 2000.

12. Oliveira DC. Análise de conteúdo temático-categorial: uma proposta de sistematização. Rev. Enferm. UERJ. 2008. 16(4): 569-76.

13. Costa TL, Oliveira DC, Formozo GA. Qualidade de vida e AIDS sob a ótica de pessoas vivendo com o agravo: contribuição preliminar da abordagem estrutural das representações sociais. Cad. Saúde Pública. 20I5. 3 I (2): 365-76.

14. Trigueiro DRSG et al. AIDS and jail: social representations of women in freedom deprivation situations. Rev Esc Enferm USP.20 I6. 50(4): 554-56I.

15. Oliveira DC et al. O significado do HIVIAIDS no processo de envelhecimento. Rev. enferm. UERJ. 20 I I. I9(3): 353-358.

16. Gomes AMT, Silva EMP, Oliveira DCO. Social representations of AIDS and their quotidian interfaces for people living with HIV. Rev Lat Am Enfermagem. 20I I. 19(3): 485-492.

17. Francisco MTR et al. Conhecimento sobre HIVIAIDS e a utilização do preservativo entre os participantes do carnaval. Revista Cubana de enfermeria [internet]. 2014 [acesso em 02 jan 2018]; 30(3). Disponível em: http://www.revenfermeria.sld.cu/index.php/enf/article/view/295.
18. Moscovici, S. O fenômeno das representações sociais. In: S. Moscovici. Representações sociais: investigação em psicologia social. 2. ed. Petrópolis:Vozes; 2007. p. 29- 109.

19. Araújo LF, Lobo CJC, Santos JVO, Sampaio AVC. Concepções psicossociais acerca do conhecimento sobre a AIDS das pessoas que vivem com HIV. Revista Colombiana de Psicologia. 2017. 26(2): 219-230.

20. Antunes L, Camargo BV, Bousfield ABS. Representações sociais e estereótipos sobre AIDS e pessoas que vivem com HIVIAIDS. Psicol. teor. prát; 20I4. I6(3): 43-57.

21. Nascimento EKS, Albuquerque LPA, Marinelli NP. História de vida de idosos com HIVIAIDS. Rev Enferm UFPE on line. 2017. I I (4): I7 16-24.

22. Silva CD, Magalhães Junior CAO, Inada JF. Psicologia Social, Representações Sociais e AIDS. Rev. Ens. Educ. Cienc. Human. 2018. 18(4):458-463.

23. Garbin CAS et al. Stigma of HIV positive expressed through veiled prejudice. DST - J bras Doenças Sex Transm. 2016. 28(2):64-66.

24. Oliveira DC. Construção e transformação das representações sociais da AIDS e implicação para os cuidados de saúde. Rev. Latino-Am. Enfermagem. 20I3. 2 I (spec):276-286.

25. Ramos ACRS, Bussinger ECA.A modelação de subjetividades e a docilização dos corpos no contexto de prevenção do HIV. In: Anais eletrônicos do Seminário Internacional fazendo gênero II e I 3 Women's Worlds Congress; 20 I7; Florianópolis.

26. Waidman MAP, Bessa JB, Silva FLC. Living with AIDS and psychological suffering. Rev Rene. 20I I. I2(I): I73-80.

27. Brito FLCB, Rosa JM. "Os leprosos dos anos 80", "câncer gay”, “castigo de Deus”: homossexualidade,AIDS e capturas sociais no Brasil dos anos 1980 a 1990. Rev. Observatório. 2018. 4(I):75I-778.

28. Nogueira VPF, Gomes AMT, Machado YY, Oliveira DC. Cuidado em saúde à pessoa vivendo com HIVIAIDS: representações sociais de enfermeiros e médicos. Rev. Enfem. UERJ. 20I5. 23(3):33I-7.

29. Ministério da Saúde (BR). Departamento de vigilância, prevenção e controle das IST, do HIVIAIDS e das hepatites virais. Protocolo clínico e diretrizes terapêuticas para manejo da infecção pelo HIV em crianças e adolescentes. Brasília, DF. 2017.

30. Oliveira FBM, Moura MEB, Araújo TME, Andrade EMLR. Qualidade de vida e fatores associados em pessoas convivendo com HIVIAIDS. Act. Paul. Enferm. 2015. 28(6):5 10-6.

31. Costa TL, Oliveira DCO, Formozo GA, Gomes AMT. Persons living with AIDS in nurses' social representations: analysis of central, contranormative and attitudinal elements. Rev Lat Am Enfermagem.20 I2. 20(6): I091 - 1099.

Recebido: 2020-0I-04 Aceito: 2020-03-02 